\title{
Spin period evolution of a recycled pulsar in an accreting binary
}

\author{
J. Wang ${ }^{1}$, C. M. Zhang ${ }^{1}$, Y. H. Zhao ${ }^{1}$, Y. Kojima ${ }^{2}$, H. X. Yin ${ }^{3}$, and L. M. Song ${ }^{4}$ \\ 1 National Astronomical Observatories, Chinese Academy of Sciences, Beijing 100012, PR China, \\ e-mail: zhangcm@bao.ac.cn \\ 2 Department of Physics, Hiroshima University, Higashi-Hiroshima 739-8526, Japan \\ 3 School of Space Science and Physics, Shandong University, Weihai 264209, PR China \\ 4 Institute of High Energy Physics, Chinese Academy of Sciences, Beijing 100049, PR China
}

Received 10 June 2010 / Accepted 7 November 2000

\section{ABSTRACT}

\begin{abstract}
We investigate the spin period evolutions of recycled pulsars in binary accreting systems. Taking both the accretion induced field decay and spin-up into consideration, we calculate their spin period evolutions influenced by the initial magnetic field strengths, initial spin periods, and accretion rates. The results indicate that the minimum spin period (or maximum spin frequency) of a millisecond pulsar (MSP) is independent of the initial conditions and accretion rate when the neutron star (NS) accretes the mass of $\sim 0.2 M_{\odot}$. The accretion torque with the fastness parameter and gravitational wave $(\mathrm{GW})$ radiation torque may be responsible for the formation of the minimum spin period (maximum spin frequency). The fastest spin frequency $(716 \mathrm{~Hz}$ ) of MSP can be inferred to associate with a critical fastness parameter of about $\omega_{\mathrm{c}}=0.55$. Furthermore, comparisons with the observational data are presented in the field period $(B-P)$ diagram.
\end{abstract}

Key words. accretion: accretion disks - stars: neutron - X-rays: binaries - pulsars: general

\section{Introduction}

Neutron stars (NSs) are usually detected as either normal pulsars (single or in a binary) with magnetic field $B \sim 10^{12} \mathrm{G}$ and spin period $P \sim 0.5 \mathrm{~s}$, or millisecond pulsars (MSPs) (half in binary) with $B \sim 10^{8.5} \mathrm{G}$ and $P \sim 20 \mathrm{~ms}$ (e.g. Bhattacharya \& van den Heuvel 1991; Lorimer 2008, see also http://relativity.livingreviews.org/Articles/ $\operatorname{lrr}-2008-8 /)$. The spin periods and surface magnetic fields assigned to these two different systems are found to span different ranges of $B-P$ values (Manchester et al. 2005; Lorimer 2008), and the updated statistical distributions of $B$ and $P$ are plotted in Fig. 1 (data from ATNF pulsar catalogue). In this figure, we notice that the distributions of both $B$ and $P$ are almost bimodal, as first suggested by Camilo et al. (1994), with a dichotomy between "normal" pulsars $(P \sim 0.1 \mathrm{~s}-10 \mathrm{~s}$ and $\left.B \sim 10^{11} \mathrm{G}-10^{13} \mathrm{G}\right)$ and $\operatorname{MSPs}(P \sim 1 \mathrm{~ms}-20 \mathrm{~ms}$ and $\left.B \sim 10^{8} \mathrm{G}-10^{9} \mathrm{G}\right)$. This abnormal phenomenon has led to the idea that radio pulsars in binary systems have been "recycled", i.e., they have been spun up owing to mass accretion during the phase of mass exchange in binaries (Alpar et al. 1982; Radhakrishnan \& Srinivasan 1982; Bhattacharya \& Srinivasan 1995). The weak magnetic field of MSP strongly supports the idea that it decays in the binary accretion phase (e.g. Taam \& van den Heuvel 1986; van den Heuvel 2004). The current widely accepted view about this phenomenon is accretion-induced evolution; i.e., NSs accreting materials from their low-mass binary companions are spun up by the angular momentum carried by the accreted materials, while the magnetic field decays (Taam \& van den Heuvel 1986; Bhattacharya \& van den Heuvel 1991; van den Heuvel 2004). The X-ray binaries are the evolutionary precursors to these "recycled" MSPs. It is evident that $B$ and $P$ of X-ray pulsars and recycled pulsars are correlated with the duration of both the accretion phase and the total amount of matter accreted (Taam \& van den Heuvel 1986; Shibazaki et al. 1989). If the NS accretes a small quantity of mass from its companion, e.g. $\sim 0.001 M_{\odot}-0.01 M_{\odot}$, a recycled pulsar with the mildly weak field and short spin period $\left(B \sim 10^{10} \mathrm{G}, P \sim 50 \mathrm{~ms}\right)$ will be formed (e.g. Francischelli et al. 2002), like PSR 1913+16 and PSR J0737-3039 (Lyne et al. 2004). The direct evidence for this recycled idea has been found in low-mass X-ray binaries (LMXB) with the accretion millisecond X-ray pulsar (AMXP), e.g. Sax J 1808.4-3658 (Wijnands \& Klis 1998), and in observing the transition link from an X-ray binary to a radio pulsar PSR J1023+0038 (Archibald et al. 2009).

At the end of the accretion phase of NS/LMXB (accreted mass $\sim 0.2 M_{\odot}$ ), the NS magnetic field may arrive at a bottom value about $10^{8} \mathrm{G}$ and spin period may reach a minimum about millisecond (Cheng \& Zhang 1998, 2000), remaining a MSP (van den Heuvel \& Bitzaraki 1995a,b). Since then, spindown due to dipole radiation has been conceived as the "standard evolution" of MSP (Urpin \& Konenkov 1997; Urpin et al. 1997, 1998). The switch-on of a radio pulsar could exist, whose radiation pressure is capable of ejecting the accreted matter out of the system and prevent any further accretion. This "radio ejection" phase in the binary will account for the formation of a MSP (Burderi et al. 2002a,b). However, the question whether all observed MSPs could be produced within this recycled framework has not been quantitatively addressed until now. The accretion induced collapse (AIC) of a white dwarf as an alternative mechanism for the formation of MSP has been studied recently (Ferrario \& Wickramasinghe 2007a,b; Hurley et al. 2009; Wickramasinghe et al. 2009), and another idea for MSP formation proposed, which claims that these objects are born with low magnetic fields (Halpern \& Gotthelf 2010).

The mechanisms for the accretion-driven field decay and spin-up in binary NSs have since then been suggested by many researchers in different proposals. The accretion flow and 

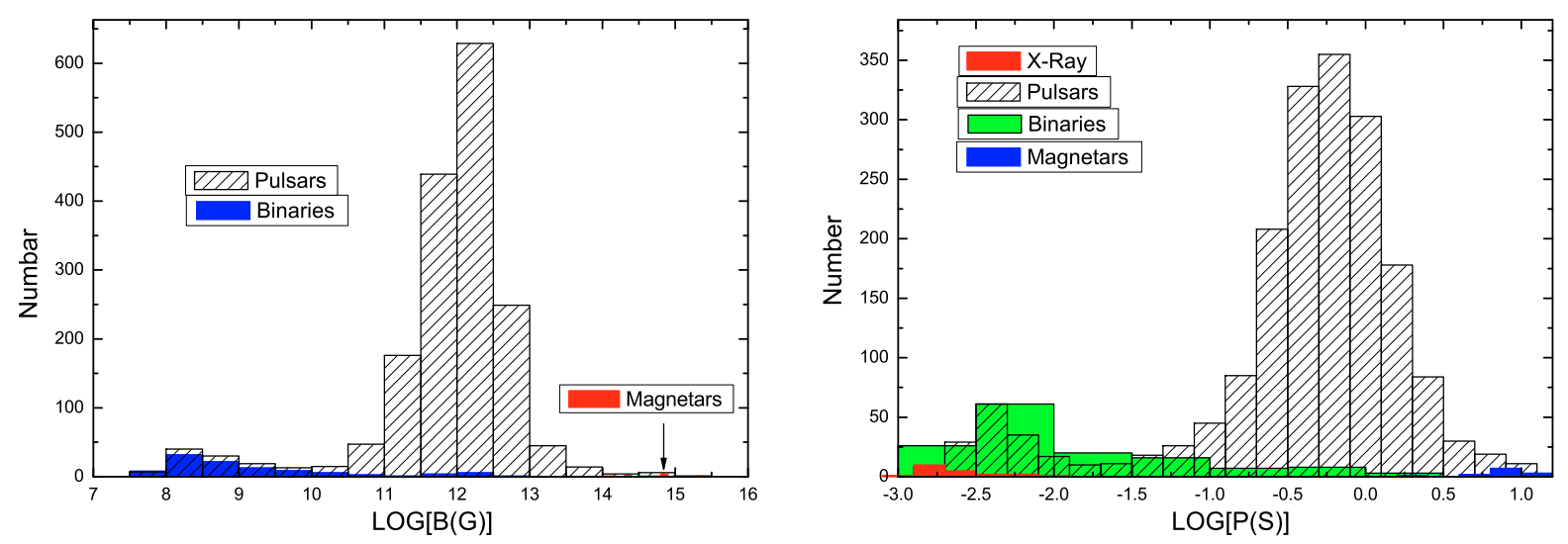

Fig. 1. Distribution of magnetic field and spin period for 1864 pulsars (data from the ATNF pulsar catalogue, see Manchester et al. 2005). Here, 141 binary pulsars and 13 magnetars (Kouveliotou 2003) are separately identified for the statistics of magnetic field and spin period. The 24 accretion millisecond X-ray pulsars discovered by RXTE (see e.g. Wijnands 2005; Lamb \& Yu 2005; Yin et al. 2007) are put in the spin period histogram.

thermal effects speed up Ohmic dissipation of NS crust currents, which accounts for the field decay and spin evolution (Geppert \& Urpin 1994; Geppert et al. 1999; Urpin \& Geppert 1995; Romani 1990). The interactions between the accreted matter and magnetic field in the course of accretion phase have been studied to account for the field decay (Melatos \& Phinney 2001; Payne \& Melatos 2004; Konar \& Bhattcharya 1999a,b; Konar \& Choudhury 2004; Lovelace et al. 2005). Not only is the total amount of accreted mass considered to influence the final field strength and minimum spin period (van den Heuvel 1995; van den Heuvel \& Bitzaraki 1994, 1995a,b), but also the influences of accretion rate may be a factor (Wijers 1997; Cumming et al. 2001; Cumming 2005; Zhang \& Kojima 2006).

Taking the accretion-induced field decay into consideration, we investigate the formation of the high spin frequency (short spin period) for a MSP and the influences on it by the initial conditions (e.g. initial spin period and initial field) and accretion rate. The spin-up torque and field evolution change their spin period and field distributions in the $B-P$ diagram from the regions of $B \sim 10^{11-13} \mathrm{G}$ and $P \sim 1 \mathrm{~s}-100 \mathrm{~s}$ to that of $B \sim 10^{8-9} \mathrm{G}$ and $P \sim 1 \mathrm{~ms}-20 \mathrm{~ms}$ as observed. This paper is organized as follows. In Sect. 2, we review the main equations that dominate the $B-P$ relation in the accretion phase. The effects of the initial conditions and accretion rate on the spin period are presented in Sect. 3 . Section 4 contains the summary and discussion.

\section{Field versus period relation}

\subsection{Description for the model}

As claimed by the accretion induced magnetic field decay model (Zhang \& Kojima 2006), if the magnetic field is sufficiently strong, e.g. $\sim 10^{12} \mathrm{G}$, and the star spin initially very slowly, the accreted matter will be channeled onto the polar patches by the field lines, where the compressed accreted matter causes the expansion of magnetic polar zone in two directions, downward and equatorward, which dilutes the magnetic flux in the polar zone. With accretion, the field decays and magnetosphere shrinks until the magnetosphere reaches the surface of the NS, where a bottom field is found to be $\sim 10^{8} \mathrm{G}$.

Based on the above model, the accretion-induced field evolution is obtained analytically with the initial field $B(t=0)=B_{0}$ (Zhang \& Kojima 2006),

$$
B=\frac{B_{\mathrm{f}}}{\left(1-[C \exp (-y)-1]^{2}\right)^{\frac{7}{4}}} .
$$

Here, we define the parameters as $y=\frac{2 \Delta M}{7 M_{\mathrm{cr}}}$, the accreted mass $\Delta M=\dot{M} t$, the crust mass $M_{\mathrm{cr}} \sim 0.2 M_{\odot}, C=1+\sqrt{1-x_{0}^{2}} \sim 2$ with $x_{0}^{2}=\left(\frac{B_{\mathrm{f}}}{B_{0}}\right)^{4 / 7}$. Also $B_{\mathrm{f}}$ is the bottom magnetic field, which is defined by the NS magnetosphere radius matching the stellar radius, i.e., $R_{\mathrm{M}}\left(B_{\mathrm{f}}\right)=R \cdot R_{\mathrm{M}}$ is defined as $R_{\mathrm{M}}=\phi R_{\mathrm{A}}$ where Alfvén radius $R_{\mathrm{A}}$ (Elsner \& Lamb 1977; Ghosh \& Lamb 1977) reads as,

$R_{\mathrm{A}}=3.2 \times 10^{8}(\mathrm{~cm}) \dot{M}_{17}^{-2 / 7} \mu_{30}^{4 / 7} m^{-1 / 7}$.

The model-dependent parameter $\phi$ is about 0.5 (Ghosh \& Lamb 1979b hereafter GL; Shapiro \& Teukolsky 1983; Frank et al. 2002), and $\dot{M}_{17}$ is the accretion rate in units of $10^{17} \mathrm{~g} / \mathrm{s}$, and $\mu_{30}$ the magnetic moment in units of $10^{30} \mathrm{Gcm}^{3}$. The mass $m=$ $M / M_{\odot}$ is in the unit of solar mass. Using the relation $R_{\mathrm{M}}\left(B_{\mathrm{f}}\right)=$ $R$, we can obtain the bottom field,

$$
B_{\mathrm{f}}=1.32 \times 10^{8}(G)\left(\frac{\dot{M}}{\dot{M}_{18}}\right)^{\frac{1}{2}} m^{\frac{1}{4}} R_{6}^{-\frac{5}{4}} \phi^{-\frac{7}{4}},
$$

where $\dot{M}_{18}=\dot{M} / 10^{18} \mathrm{~g} / \mathrm{s}$ and $R_{6}=R / 10^{6} \mathrm{~cm}$. As claimed by Inogamov \& Sunyaev (1999), in the accretion process, a boundary layer forms between the innermost disk and magnetosphere due to the transition for rotating velocity of plasma from Keplerian to the spin velocity of NS. When the accretion rate increases, this layer may spread (Inogamov \& Sunyaev 1999), and as a result more matter is accreted to the polar gap and diffuses to the entire surface of the NS. This effect will influence the field decay efficiency of the model by Zhang \& Kojima (2004), since some spreading matter contributes little to the field lines dragging on the polar patches. Moreover, such a spreading will expand the polar cap area, which makes the polar cap occupy the entire NS surface, while the magnetosphere does not reach the NS radius. The bottom field of NS is determined by the condition that the magnetosphere equals the NS radius (Zhang \& Kojima 2004). If there is no field decay while the magnetosphere radius is bigger than NS radius, then the modified bottom field by considering the spreading of the polar cap will be bigger than the one obtained for the ideal frozen plasma in the polar cap zone. Namely, the magnetic field of recycled NS is slightly bigger than the ideal value of the model by Zhang \& Kojima (2004). 
To study the spin evolution of NS in accretion phase, we employ the formula for accretion-induced spin-up given by GL,

$$
\begin{aligned}
-\dot{P}= & 5.8 \times 10^{-5}\left[\left(\frac{M}{M_{\odot}}\right)^{-\frac{3}{7}} R_{6}^{\frac{12}{7}} I_{45}^{-1}\right] \\
& \times B_{12}^{\frac{2}{7}}\left(P L_{37}^{\frac{3}{7}}\right)^{2} n\left(\omega_{\mathrm{s}}\right) \mathrm{s} \mathrm{yr}^{-1},
\end{aligned}
$$

where we define the parameters, the surface field $B_{12}=$ $B / 10^{12} \mathrm{G}$, the moment of inertia $I_{45}=I / 10^{45} \mathrm{~g} \mathrm{~cm}^{2}$, the X-ray brightness $(L=G M \dot{M} / R) L_{37}$ in units of $10^{37} \mathrm{erg} / \mathrm{s}$. The dimensionless parameter $n\left(\omega_{\mathrm{s}}\right)$ is the fastness parameter, which is explained in the following section.

\subsection{Fastness parameter}

For a slowly rotating magnetic NS, the rotation of the star couples to the orbital motion of the disk matter at the Alfvén radius when accretion flow is approximately radial, so the limit of angular velocity $\left(\Omega_{\mathrm{S}}=2 \pi / P\right)$ for a NS can reach the Keplerian velocity $\left(\Omega_{\mathrm{k}}\left(R_{\mathrm{M}}\right)=\sqrt{G M / R_{\mathrm{M}}^{3}}\right)$. In general, the spin velocity is always less than the orbital velocity, i.e. $\Omega_{\mathrm{s}} \ll \Omega_{\mathrm{k}}\left(R_{\mathrm{M}}\right)=$ $\sqrt{G M / R_{\mathrm{M}}^{3}}$. Therefore, the relative importance of stellar rotation can be described by the ratio parameter of the angular velocities (Elsner \& Lamb 1977; Ghosh \& Lamb 1977; Li \& Wang 1996, 1999; Shapiro \& Teukolsky 1983),

$\omega_{\mathrm{s}} \equiv \frac{\Omega_{\mathrm{s}}}{\Omega_{\mathrm{k}}\left(R_{\mathrm{M}}\right)}=1.35\left[\left(\frac{M}{M_{\odot}}\right)^{-2 / 7} R_{6}^{15 / 7}\right] B_{12}^{6 / 7} P^{-1} L_{37}^{-3 / 7}$,

which plays a significant role in our entire understanding of accretion to the rotating magnetic NSs.

To calculate the accretion torque that acts on a magnetic NS accreting matter from a disk, GL introduced a dimensionless accretion torque $n\left(\omega_{\mathrm{s}}\right)$ that primarily depends on the fastness parameter. A simple expression for $n\left(\omega_{\mathrm{s}}\right)$ is given by GL,

$n\left(\omega_{\mathrm{s}}\right)=1.4 \times\left(\frac{1-\omega_{\mathrm{s}} / \omega_{\mathrm{c}}}{1-\omega_{\mathrm{s}}}\right)$

where $\omega_{\mathrm{c}}$ is the critical value. For a slowly rotating $\operatorname{star}\left(\omega_{\mathrm{s}} \ll 1\right)$, GL found that the dimensionless function $n\left(\omega_{\mathrm{s}}\right)$ decreases with $\omega_{\mathrm{s}}$ and becomes negative for $\omega_{\mathrm{s}}>\omega_{\mathrm{c}}$ if $n\left(\omega_{\mathrm{s}}\right) \sim 1.4$. In addition, GL also stresses $\omega_{\mathrm{c}} \sim 0.35$ for their model. However, the subsequent work (Ghosh \& Lamb 1992) indicates that $\omega_{\mathrm{c}}$ is unlikely to be less than 0.2 , but it could be as large as 0.9 . By the aid of this dimensionless parameter, we can establish the relation between the total torque on the star and the torque communicated to the star by the magnetic field lines that thread the inner transition zone.

In the following subsections, we study the spin evolution of NS in a binary system, where the influence of fastness is included.

\section{Spin evolution of accreting NS}

In this section, we investigate the spin period evolution of accreting NSs by solving Eqs. (1), and (4)-(6) with different initial conditions and accretion rates, where we set the usual parameters for NS, e.g. $m=1.4, R_{6}=1.5, \phi=0.5$, and $M_{\text {cr }}=0.2 M_{\odot}$.

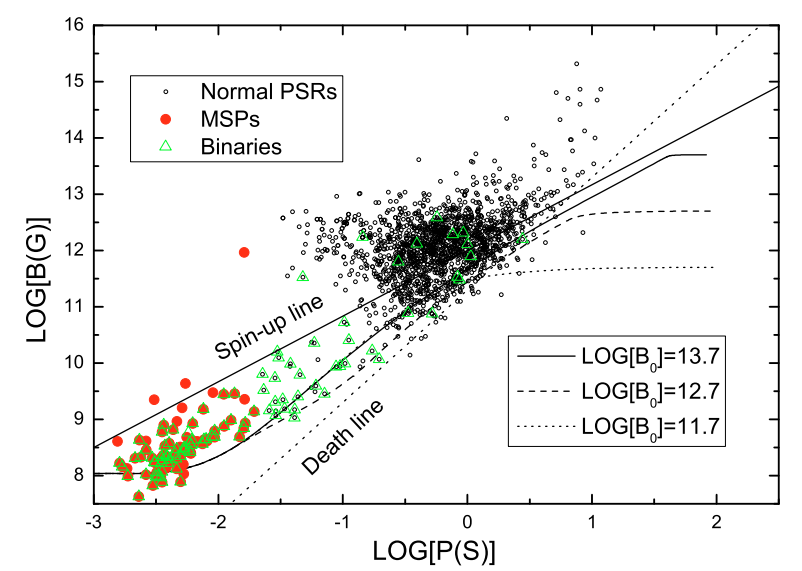

Fig. 2. The diagram of the magnetic field versus spin period for pulsars. The spin up line represents the minimum spin period to which such a spin up may proceed in an Eddington-limited accretion, while the "death line" corresponds to a polar cap voltage below which the pulsar activity is likely to switch off (Bhattacharya \& van den Heuvel 1991). The evolutionary tracks in $B-P$ diagram are plotted with different initial magnetic field strengths, but with same initial spin period $\left(P_{\mathrm{o}}=100 \mathrm{~s}\right)$ and the same accretion rate $\dot{M}_{17}$; the solid, dashed, and dotted lines denote the numerical solutions for Eqs. (1), (4)-(6) with initial field $B_{\mathrm{o}}=5 \times 10^{13} \mathrm{G}, 5 \times 10^{12} \mathrm{G}$ and $5 \times 10^{11} \mathrm{G}$, respectively.

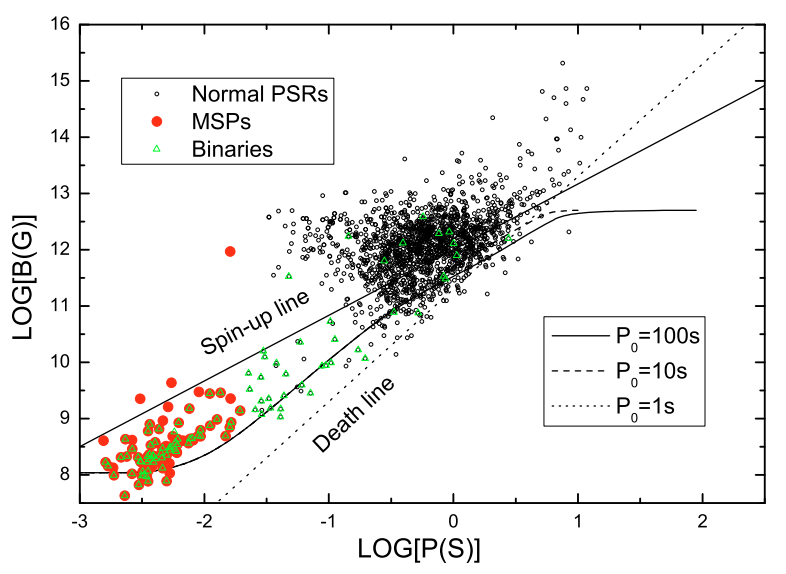

Fig. 3. The same meaning as Fig. 2 with different initial spin periods $\left(P_{\mathrm{o}}=1 \mathrm{~s}-\right.$ dotted line, $10 \mathrm{~s}-$ dashed line, $100 \mathrm{~s}$ - solid line $)$ at the fixed initial magnetic field $\left(B_{\mathrm{o}}=5 \times 10^{12} \mathrm{G}\right)$ and the same accretion rate $\dot{M}_{17}$.

\subsection{Influence by initial conditions}

We consider the spin evolution for a wide range of initial conditions (initial magnetic fields and initial spin periods): (1). varying the initial magnetic field $5 \times 10^{11} \mathrm{G}, 5 \times 10^{12} \mathrm{G}$, and $5 \times 10^{13} \mathrm{G}$, while setting spin period and accretion rate as 100 s and $\dot{M}_{17}$, respectively (Fig. 2); (2). varying the initial spin period $1 \mathrm{~s}, 10 \mathrm{~s}$ and $100 \mathrm{~s}$ with a certain field $\left(5 \times 10^{12} \mathrm{G}\right)$ and constant accretion rate $\left(\dot{M}_{17}\right)$ (Fig. 3 ).

We classify the samples as normal pulsars, MSPs, and binaries. From the figures, we can see that most MSPs are consistent with the binary pulsars, while only a few binaries fall in the same region of the normal pulsars that have a magnetic field $\sim 10^{11} \mathrm{G}$. Recycled pulsars are expected to be found only to the right of the spin-up line and to the left of the "death line" (Bhattacharya \& van den Heuvel 1991), which is indeed shown to be the case in these figures (Figs. 2, 3) for almost all the binaries and MSPs. 
This strongly supports the view that the MSPs have been recycled (Alpar et al. 1982; Radhakrishnan \& Srinivasan 1982). First, with a small amount of mass transferred, the NS is spun up from the death valley with a long period and strong field, e.g. the NS in HMXBs $\left(B \sim 10^{12} \mathrm{G}, P \sim 10 \mathrm{~s}\right)$ like Her X-1 $\left(P=1.24 \mathrm{~s}, B=3 \times 10^{12} \mathrm{G}\right.$, see van der Meer et al. 2007; Liu et al. 2007). With the long-lived accretion phase, the binary accepts sufficient mass from its companion and the NS yields a substantial field decay, as in the case of MSPs (Bhattacharya \& van den Heuvel 1991), e.g., the fastest known pulsar PSR J17482446 ( $B \sim 10^{8} \mathrm{G}, P=1.4 \mathrm{~ms}$, see Hessels et al. 2006).

As shown in Fig. 2, three $B-P$ tracks with the different initial field values begin to follow one track when the spin period evolves to the hundred millisecond regime, and here the system accretes $\sim 0.01 M_{\odot}$. After the system accretes $\sim 0.2 M_{\odot}$, the field reaches $\sim 10^{8} \mathrm{G}$, the lowest value, and the spin period enters the millisecond regime, which is inferred to corresponding to the condition $\omega_{\mathrm{s}}=\omega_{\mathrm{c}}$. The bottom spin period or maximum spin frequency can be obtained by

$v_{\mathrm{SMax}}=v_{\mathrm{k}}\left(R_{\mathrm{M}}=R\right) \omega_{\mathrm{c}}$

where $v_{\mathrm{k}}\left(R_{\mathrm{M}}=R\right)=1300(\mathrm{~Hz})\left(M / 1.4 M_{\odot}\right)^{1 / 2}(R / 15 \mathrm{~km})^{-3 / 2}$ is the maximum spin frequency at the stellar surface. If the critical fastness parameter is $\omega_{\mathrm{c}}=0.55$, then the maximum spin frequency can be as high as $715 \mathrm{~Hz}$, which is similar to the fastest spin frequency of MSP observed $(716 \mathrm{~Hz})$. The lower the critical fastness, the lower the final spin frequency achieved.

For our numerical calculation, we take the effect of fastness parameter into consideration. If the critical fastness parameter is set at 0.9 , the numerical solutions for Eqs. (1), (4)-(6) suggest that the evolutionary curves in the $B-P$ diagram are a little below the spin period equilibrium line. However, the effect of the fastness parameter is significant when the $B-P$ evolutionary track is close to the equilibrium period line (spin-up line). This indicates that the spin angular velocity of the star tends to reach the Keplerian angular velocity at the inner edge of the accretion disk, but never exceeds this angular velocity. In fact, the primary effect of the fastness parameter is to force the evolution curves back to or below the equilibrium period line and ensure that the evolutionary track cannot go beyond this line.

From these tracks, we can see that, despite the differences for initial fields and initial spin periods, the NSs can approach a certain minimum spin period and bottom field after accreting up to $\sim 0.2 M_{\odot}$. If the accreting phase is over, a recycled pulsar can be found in the region of $B-P$ diagram with $B \sim 10^{8-9} \mathrm{G}$ and $P \sim 10 \mathrm{~ms}$, forming a MSP. On account of the existence of minimum spin period and bottom field (e.g. $B \sim 10^{8} \mathrm{G}$ ), the $P$ distribution and $B$-distribution imply that the MSPs accumulate close to the region of low field and short period, which is an explanation of the bimodal distributions for all pulsars, as shown in Fig. 1. In short, for LMXBs, the different initials give the similar final value of spin period, which is insensitive to the initial conditions.

\subsection{Influence by accretion rate}

In this part, we study the influence of accretion rates on the spin period evolution. We set the accretion rate as $\dot{M}_{16}, \dot{M}_{17}$ and $\dot{M}_{18}$ at the fixed magnetic field $\left(5 \times 10^{12} \mathrm{G}\right)$ and spin period $(100 \mathrm{~s})$ (see Fig. 4). From Fig. 4, we find that, with different accretion rates, the spin evolution follows different tracks. If we set the critical fastness parameter as 0.9, the tracks with Eddington accretion rate $\left(\dot{M}_{18}=1\right)$ matches the evolution of most recycled pulsars closely, but the track with $\dot{M}_{16}$ lies below the "death

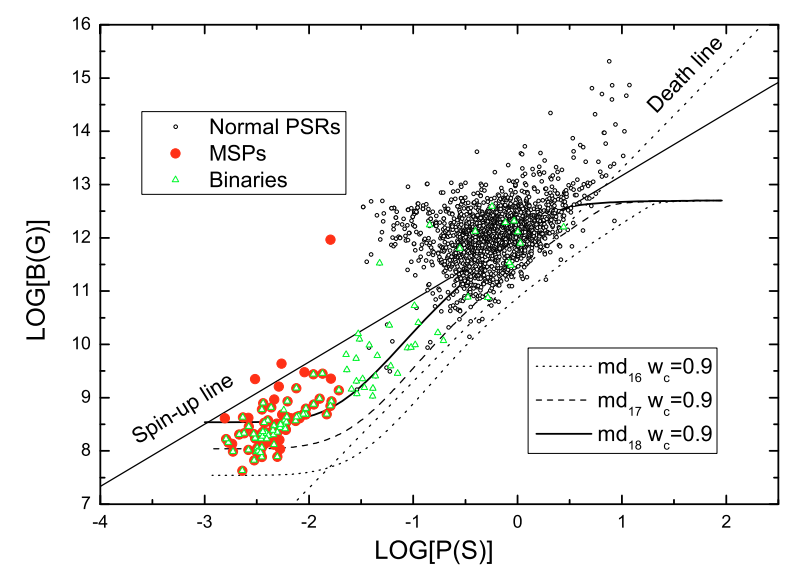

Fig. 4. The same meaning as Fig. 2 with different accretion rates, at the fixed initial magnetic field $\left(B_{\mathrm{o}}=5 \times 10^{12} \mathrm{G}\right)$ and initial spin period $\left(P_{\mathrm{o}}=\right.$ $100 \mathrm{~s}$ ), from the top to bottom, $\dot{M}_{18}$ - solid line, $\dot{M}_{17}$ - dashed line, and $\dot{M}_{16}$ - dotted line, corresponding to the bottom fields of $\sim 10^{7.5} \mathrm{G}$, $\sim 10^{8} \mathrm{G}$, and $\sim 10^{8.5} \mathrm{G}$, respectively. The critical fastness parameter is set to be $\omega_{\mathrm{c}}=0.9$.

line". Moreover, the curves with a high accretion rate and high critical fastness have the tendency to approach the spin-up line.

For a comparison of the magnetic field and spin period distributions between theory and observation, we plot the statistical distributions of magnetic field and spin period obtained from calculating theoretical model with various initial conditions (see caption of Fig. 5). One hundred samples of the final $B$-field and final spin period $(B, P)$ were done, which are plotted to compare the observed data of binary pulsars as shown in Fig. 5, where we notice bimodal distributions of magnetic field and spin period of recycled pulsars (initial distributions of $B$ and $P$ in the right parts of plots compared to the final evolved values in the left parts of plots). As compared to the observational distributions of binary pulsars, we notice that both histograms for the MSPs (left parts of Fig. 5), theoretical and observed data, are very similar. For the spin period, more theoretical data than the observed values occurs at the regimes close to millisecond, which may imply that more MSPs may not evolve to their minimum spin period of about millisecond.

\subsection{Spin period versus accretion mass $(P-\Delta M)$}

To investigate the spin period evolution with accretion mass, we considered the influences by initial conditions and accretion rate and plot the evolutionary curves in $P-\Delta M$ diagrams (see Fig. 6). As a detailed illustration, Fig. 6 implies the following results. (1). After accreting a given mass (e.g. $\sim 0.01 M_{\odot}$ ), the spin period evolution seems independent of the initial magnetic field. (2). The influence on $P-\Delta M$ by the initial spin period on the spin evolution exists when the accreting mass is no more than $\Delta M \sim 0.001 M_{\odot}$ and disappears when the accreted mass is much more than $\Delta M \sim 0.001 M_{\odot}$. When a small amount of mass $\left(\sim 0.001 M_{\odot}\right)$ is transferred, the spin period changes mildly (Fig. 6), which may yield a HMXB with the spin period of some seconds, such as Her X-1 (van der Meer et al. 2007) and Vela X-1 (Quaintrell et al. 2003). After a conveniently long time, with the weight of material accreted $\sim 0.01 M_{\odot}$, NS is spun up to a shorter period $(P \sim 100 \mathrm{~ms})$, which corresponds to the system of DNS, e.g. PSR 1913+16 (Breton 2009). The binaries with the longer accretion phases, e.g. LMXBs, will accrete enough mass $\left(\Delta M \sim 0.1 M_{\odot}\right)$ from their companions to yield a lower magnetic field and shorter spin period $(P \sim 20 \mathrm{~ms})$, as in the case 

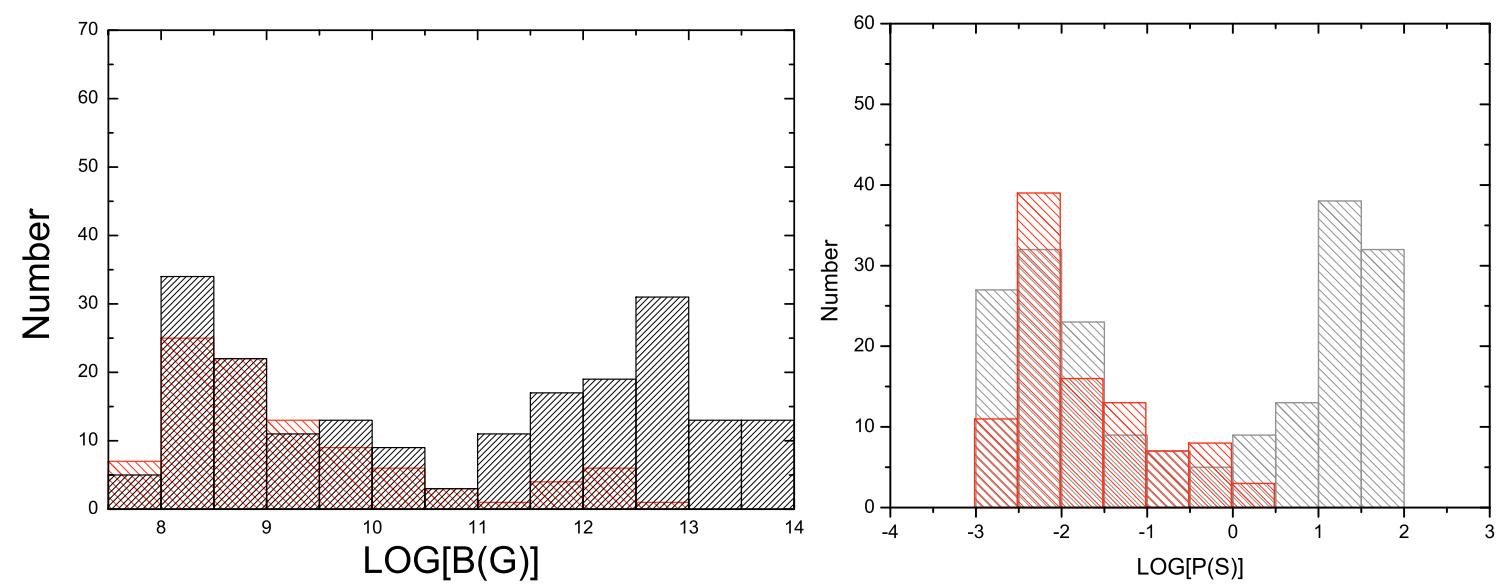

Fig. 5. Plots of histograms for spin period and magnetic field obtained from calculations of the theoretical model and for the observed binary pulsars (red-shaded). In theoretical calculations, we assume that the initial $B$-field follows a Gaussian distribution, centered at $5 \times 10^{12} \mathrm{G}$ and ranging from $5 \times 10^{10} \mathrm{G}$ (e.g. Hartman et al. 1997) to $10^{14} \mathrm{G}$ (e.g. Kaspi 2010). As for the initial spin period, it is assumed to be a Gaussian distribution, centered at $30 \mathrm{~s}$ and ranging from $0.5 \mathrm{~s}$ to $100 \mathrm{~s}$. The different accretion rates are set from $\dot{M}_{16}$ to $\dot{M}_{18}$. The theoretically evolved $B$-field and spin period values occurred in the left parts of the plots, which are similar to the observed distributions.
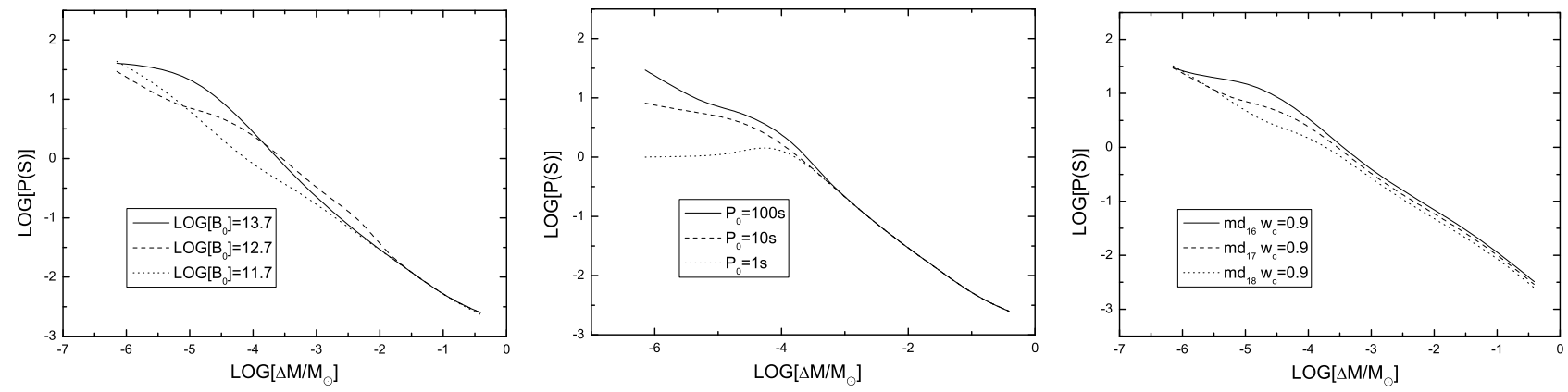

Fig. 6. Plots of spin period versus accretion mass with different conditions, i.e., initial magnetic fields (left), initial spin periods (middle) and accretion rates (right), where the critical fastness parameter is set to be $\omega_{\mathrm{c}}=0.9$ in calculation.

of MSPs, e.g. SAX J 1808.4-3658 (Wijnands \& van der Klis 1998; Wijnands 2005) and PSR J 1748-2446 (Lorimer 2008). (3). At the beginning of accretion, NSs with different accretion rates follow different curves in the $P-\Delta \mathrm{M}$ plane. After accreting $\sim 0.01 M_{\odot}$, the spin period follows approximately the same track with a different accretion rate and goes to the same minimum period. Therefore, NSs in LMXBs for the Z source with Eddington luminosity and Atoll source with less luminosity, e.g. 1\% Eddington accretion rate (Hasinger \& van der Klis 1989 ), should have the similar spin frequencies while accreting $\sim 0.2 M_{\odot}$.

In addition, it is noticed that the initial conditions $\left(B_{0}\right.$ and $\left.P_{0}\right)$ and accretion rate have little influence on the $P-\Delta \mathrm{M}$ plot after the system accretes up to $0.01 M_{\odot}$; in other words, the spin periods of NS/LMXBs should be primarily related to how much mass is accreted.

\section{Summary and discussion}

Considering the influences exerted by the different initial magnetic fields, initial spin periods, and the accretion rate, we have investigated the evolutionary tracks of recycled pulsar in $B-P$ diagram. The main results are listed below.

(1). From the evolutionary tracks in $B-P$ diagram, we find that the magnetic field of NS decays with accreting material, and the spin-up evolution proceeds at the same time. The primary effect of the fastness parameter is to force the evolutionary curves to reside below the equilibrium period line. The critical fastness $\left(\omega_{\mathrm{c}}\right)$ can infer the final spin period of recycled $\mathrm{NS}$ (maximum spin frequency), for instance, $\omega_{\mathrm{c}}=0.5$ will correspond to a spin of $650 \mathrm{~Hz}(1.54 \mathrm{~ms})$, which is near the highest observed value of MSP and AMXP.

(2). The influences of the initial magnetic field, spin period and accretion rate are tested. The evolutionary curves show that the spin period (frequency) decreases (increases) with the accretion, and the minimum spin period is insensitive to these two initial conditions when NS is spun up to $P \sim 10 \mathrm{~ms}$ $(v \sim 100 \mathrm{~Hz})$ after NS accreting $\sim 0.2 M_{\odot}$.

(3). It is found from the B-P diagram that a few observed MSPs are located above the "spin up line", while the trajectories based on our model always lie below this line. First, owing to the Ohmic dissipation, the buried field of MSP may reemerge after accretion, which can account for a slight increase in the field of the star (e.g. Young \& Chanmugam 1995; Bhattacharya 2008). Second, the magnetic model we used here is based on the "dilution of polar magnetic flux" due to accretion, which is idealized. There are numerous plasma instabilities, e.g., the Rayleigh-Taylor instability at the disk-magnetosphere interface because of the high-density disk matter being supported against by the lowdensity magnetosphere plasma, and the Kelvin-Helmholtz instability because of the discontinuity in the angular velocity of matter at the boundary (Ghosh \& Lamb 1979a), and 
these may result in the penetration of the magnetosphere, prying the field lines aside and azimuthally wrapping the field lines by the disk matter (e.g. Romanova 2008; Kulkarni \& Romanova 2008), which in turn may modify the field strength evolution and then perturb the spin evolution.

(4). The formation of the fastest spin frequency of MSP is an interesting topic (Lorimer 2008; Chakrabarty et al. 2003; Chakrabarty 2005). The known fastest radio pulsar is PSR J1748-2446ad (Ter 5) at $716 \mathrm{~Hz}$ (Hessels et al. 2006), whereas the highest AMXP spin frequency in LMXBs is $619 \mathrm{~Hz}$ (e.g. Wijnands 2005). However, the recent report of $1122 \mathrm{~Hz}$ burst oscillation frequency, interpreted as a spin frequency, in XTE J 1739-285 (Kaaret et al. 2007) has been reported but not yet confirmed on account of the statistical significance (actual significance is only $3 \sigma$ ). Now the detected spin is less than the believed breaking spin frequency of pulsar of $\sim 1000 \mathrm{~Hz}$ (Lattimer \& Prakash 2004), so what causes the special maximum spin frequency of MSP?

The spin evolution is affected by the accretion torque, which consists of two parts (Ghosh 1995; Frank et al. 2002): the stresses associated with matter accreting from the inner edge of the disk $\left(N_{0} \equiv \dot{M}\left(G M R_{\mathrm{M}}\right)^{\frac{1}{2}}\right)$ and that associated with the magnetic field coupling the star with the disk. The total torque $N$ can be conveniently expressed in terms of $N_{0}$ and the dimensionless torque. The spin-down torques that balance the spin-up torque will also contribute to forming the maximum spin frequency. Slow rotators $\left(\omega_{\mathrm{s}}<\omega_{\mathrm{c}}\right)$ are spun up $(n>0)$ by this accretion torque, where $\omega_{\mathrm{s}}<1$ is presumed, since for $\omega_{\mathrm{s}}>1$ the steady accretion is of course impossible. Here, a $\omega_{\mathrm{c}}$ of approximately $\sim 0.3-0.5$ is the critical fastness at which the torque changes direction, thus the maximum spin frequency $v_{\mathrm{s}}=2 \pi \Omega_{\mathrm{s}}=\omega_{\mathrm{c}} v_{\mathrm{k}} \sim$ $(0.3-0.5) v_{\mathrm{k}}$, where $v_{\mathrm{k}}=2 \pi \Omega_{\mathrm{k}}$. The observed maximum upper $\mathrm{kHz}$ QPO frequency is $1330 \mathrm{~Hz}$ (van der Klis 2000; Zhang et al. 2006), which is interpreted as the Keplerian frequency $v_{K}$ of inner disk orbit, so this implies a maximum spin frequency of $\sim 400-665 \mathrm{~Hz}$ which is consistent with observations. To limit the occurrence of high spin of MSP over $1000 \mathrm{~Hz}$, the braking torques were considered. Electromagnetic braking torque can brake the stellar spin (Ghosh 1995, 2006), which is usually expressed in the form $n \propto \mu^{2}\left(\Omega_{\mathrm{S}} / c\right)^{3}$, where $\mu$ is the magnetic dipole moment of the star. In NS/LMXB, the mass quadrupole can occur on account of the accretion, which arises the gravitational wave (GW) radiation (Bildsten 1998; Melatos 2007; Melatos \& Payne 2005; Melatos \& Peralta 2010; Ghosh 2006; Vigelius et al. 2008; Watts et al. 2009). The angular momentum is lost from the emission of GW which may spin the NS down. With accretion, the gravitational wave and accretion torques balance, but the total torque is actually negative because the gravitational wave torque increases with accretion, hence, a maximum spin frequency of NS might arise with several hundred Hertz.

Acknowledgements. We are grateful for the discussions with G. Hasinger, M. Mendez, and T. Belloni. This work is supported by the National Natural Science Foundation of China (NSFC 10773017) and the National Basic Research Program of China (2009CB824800).

\section{References}

Archibald, A. M., Stairs, I. H., \& Ransom, S. M., et al. 2009, Science, 324, 1411 Alpar, M. A., Cheng, A. F., \& Ruderman, M. A., et al. 1982, Nature, 300, 728 Bhattacharya D. 2008, Proceedings of A Decade of Accreting Millisecond X-ray Pulsars, ed. R. Wijnands et al., AIPC, 1068, 137

Bhattacharya, D., \& van den Heuvel, E. P. J. 1991, Phys. Rep., 203, 1

Bhattacharya, D., \& Srinivasan, G. 1995, in X-ray Binaries, ed. W. H. G. Lewin,

J. van Paradijs, \& E. P. J. van den Heuvel (Cambridge University Press)
Bildsten, L. 1998, ApJ, 501, L89

Breton R. P. 2009, PhD Thesis [arXiv: 0907.2623]

Burderi, L., D’Antona, F., Menna, M. T., di Salvo, T., \& Robba, N. 2002a, Mem. Soc. Astron. Ital., 73, 1072

Burderi, L., D’Antona, F., \& Burgay, M. 2002b, ApJ, 574, 325

Camilo, F., Thorsett, S. E., \& Kulkarni, S. R. 1994, ApJ, 421, L15

Chakrabarty, D., Morgan, E. H., Muno, M. P., et al. 2003, Nature, 424, 42

Chakrabarty, D. 2005, ASP Conf. Ser., 328, 279, to appear in Binary Radio Pulsars, eds. F.A.

Cheng, K. S., \& Zhang, C. M. 1998, A\&A, 337, 441

Cheng K. S., \& Zhang, C. M. 2000, A\&A, 361, 1001

Cumming, A., Zweibel, E. G., \& Bildsten, L. 2001, ApJ, 557, 958

Cumming, A. 2005, ASP Conf. Ser., 328, 311

Ferrario, L., \& Wickramasinghe, D. T. 2007a, AIP Conf., 968, 188

Ferrario, L., \& Wickramasinghe, D. T. 2007b, AIP Conf., 968, 194

Elsner, R. F., \& Lamb, F. K. 1977, ApJ, 215, 897

Francischelli, G. J., Wijers, R. A. M. J., \& Brown, G. E. 2002, ApJ, 565, 471

Frank, J., King, A., \& Raine, D. J. 2002, Accretion Power in Astrophysics, Cambridge, UK

Geppert, U., \& Urpin, V. 1994, MNRAS, 271, 490

Geppert, U., Page, D., \& Zannias, T. 1999, A\&A, 345, 847

Ghosh, P., \& Lamb, F. K. 1977, ApJ, 217, 578

Ghosh, P., \& Lamb, F. K. 1979a, ApJ, 232, 259

Ghosh, P., \& Lamb, F. K. 1979b, ApJ, 234, 296

Ghosh, P., \& Lamb, F. K. 1992, in X-ray Binaries and Recycled Pulsars, ed. E.

P. J. van den Heuvel, \& S. A. Rappaport (Dordrecht: Kluwer), 487

Ghosh, P. 1995, JApA, 16, 289

Ghosh, P. 2006, Rotation and Accretion Powered Pulsars, World Scientific, India

Hartman, J. W., Portegies Zwart, S., \& Verbunt, F., et al. 1997, 325, 1031

Hasinger, G., \& van der Klis, M. 1989, A\&A, 225, 79

Halpern, J. P., \& Gotthelf, E. F. 2010, ApJ, 709, 436

Hessels, J. W., Ransom, S. M., \& Stairs, I. H., et al. 2006, Science, 311, 1901

Hurley, J. R., Tout, C. A., Wickramasinghe, D. T., Ferrario, L., \& Kiel, P. D. 2009, MNRAS, 402, 1437

Inogamov, N. A., \& Sunyaev, R. A. 1999, AstL., 25, 269

Kaaret, P., Prieskorn, Z., in't Zand, J., et al. 2007, ApJ, 657, L97

Kaspi, V. M. 2010, PNAS, 107, 7147

Konar, S., \& Bhattacharya, D. 1999a, MNRAS, 303, 588

Konar, S., \& Bhattacharya, D. 1999b, MNRAS, 308, 795

Konar, S., \& Choudhury, A. 2004, MNRAS, 348, 661

Kouveliotou, C. 2003, AAS, 203, 7501

Kulkarni, A. K., \& Romanova, M. M. 2008, MNRAS, 386, 673

Lamb, F. K., \& Yu, W. 2005, in Spin rates and magnetic fields of millisecond pulsars, in Binary Radio Pulsars, ed. F. A. Rasio, \& I. H. Stairs, ASP Conf. Ser., 328, 299

Lattimer, J. M., \& Prakash, M. 2004, Science, 304, 536

Li, X. D., \& Wang, Z. R. 1996, A\&A, 307, L5

Li, X. D., \& Wang, Z. R. 1999, ApJ, 513, 845

Liu, Q. Z., van Paradijs, J., \& van den Heuvel, E. P. J. 2007, A\&A, 469, 807

Lorimer, D. R. 2008, Living Rev. Relativity, 11, 8

Lovelace, R. V., Romanova, M. M., \& Bisnovatyi-Kogan, G. S. 2005, ApJ, 625, 957

Lyne, A. G., Burgay, M., \& Kramer, M., et al.2004, Science, 303, 1153

Manchester, R. N., Hobbs, G. B., Teoh, A., \& Hobbs, M. 2005, AJ, 129, 1993

Melatos, A. 2007, AdSpR, 40, 1472

Melatos, A., \& Phinney, E. S. 2001, PASA, 18, 421

Melatos, A., \& Payne, D. 2005, ApJ, 623, 1044

Melatos, A., \& Peralta, C. 2010, ApJ, 709, 77

Payne, D., \& Melatos, A. 2004, MNRAS, 351, 569

Quaintrell, H., Norton, A. J., \& Ash, T. D. C. et al. 2003, A\&A, 401, 313

Radhakrishnan, V., \& Srinivasan, G. 1982, Curr. Science, 51, 1096

Romani, G. M. 1990, Nature, 347, 741

Romanova, M. M., Kulkarni, A. K., \& Lovelace, R. V. E. 2008, ApJ, 673, L171

Shapiro, S. L., \& Teukolsky, S. A. 1983, Black Holes, White Dwarfs and Neutron Stars (New York: Wiley)

Shibazaki, N., Murakami, T., Shaham, J., \& Nomoto, K. 1989, Nature, 342, 656

Taam, R. E., \& van den Heuvel, E. P. J. 1986, ApJ, 305, 235

Urpin, V., \& Geppert, U. 1995, MNRAS, 275, 1117

Urpin, V., \& Konenkov, D. 1997, 284, 741

Urpin, V., Geppert, U., \& Konenkov, D. 1997, MNRAS, 295, 907

Urpin, V., Geppert, U., \& Konenkov, D. 1998, A\&A, 331, 244

van den Heuvel, E. P. J. 1995, JA\&A, 16, 255

van den Heuvel, E. P. J. 2004, Science, 303, 1143

van den Heuvel, E. P. J., \& Bitzaraki, O. 1994, MmSAI, 65, 237

van den Heuvel, E. P. J., \& Bitzaraki, O. 1995a, A\&A, 297, L41

van den Heuvel, E. P. J., \& Bitzaraki, O. 1995b, in The Lives of the Neutron

Stars (Dordrecht: Kluwer Academic Publishers)

van der Klis, M. 2000, ARA\&A, 38, 717 
J. Wang et al.: Spin period evolution of a recycled pulsar in an accreting binary

van der Meer, A., Kaper, L., van Kerkwijk, M. H., Heemskerk, M. H. M., \& van den Heuvel, E. P. J. 2007, A\&A, 473, 523

Vigelius, M., Payne, D., \& Melatos, A. 2008, Proceedings of the 11th Marcel Grossmann Meeting on General Relativity, World Scientific [arXiv:0811.2031]

Watts, A. L., et al. 2009, MNRAS, 389, 839

Wickramasinghe, D. T., Hurley, J. R., Ferrario1, L., Tout, C. A., \& Kiel, P. D. 2009, JPhCS., 172, 2037
Wijnands, R., \& van der Klis, M. 1998, Nature, 394, 344

Wijnands, R. 2006, Trends in Pulsar Research, ed. J. A. Lowry (New York, USA:

Science Publishers, Inc.), 53

Wijers, R. A. M. J. 1997, MNRAS, 287, 607

Yin, H. X., Zhang, C. M., \& Zhao, Y. H. 2007, A\&A, 471, 381

Young, E. J., \& Chanmugam, G. 1995, ApJ, 442, L53

Zhang, C. M., \& Kojima, Y. 2006, MNRAS, 366, 137

Zhang, C. M., Yin, H. X., Zhao, Y. H., et al. 2006, MNRAS, 366, 1373 